\title{
Application of spin-orbit-coupling induced magnetic field effects for probing the dynamics of photoinduced charge separation and recombination
}

\author{
U E STEINER*, W HAAS, H -J WOLFF and D BÜRSSNER \\ Fakultät für Chemie, Universität Konstanz, Germany
}

\begin{abstract}
In this paper we present two examples demonstrating how magnetic field effects can be utilized for a quantitative exploration of mechanistic details of the behaviour of short-lived redox intermediates in photoelectron transfer reactions with excited triplet states. This magnetokinetic approach takes advantage of the spin memory present in the intermediates (exciplexes, correlated radical pairs) which controls the competition between spin-allowed formation of free redox products and spin-forbidden backward electron transfer regenerating the ground state reactants. In particular we demonstrate that the strong spin-orbit coupling exhibited by a heavy atom substituent or by heavy transition metal centers can be very useful for inducing and exploiting such diagnostic magnetic field effects.
\end{abstract}

Keywords. Photoinduced electron transfer, triplet exciplexes; radical pairs; magnetic field effects; spin-orbit coupling; spin-forbidden processes.

\section{Introduction}

During the last two decades various types of experiments have been developed that demonstrate and exploit the delicate role of electron spin in the control of reaction rates and yields of chemical reactions involving intermediates with unpaired couples of electron spins as in radical pairs or in excited triplet states (Salikhov et al 1984; Steiner and Ulrich 1989). From such experiments and their underlying theories a coherent field of research that may be called spin chemistry (Hayashi 1990) has emerged. One of its characteristic methods utilizes the effect of static magnetic fields on the rates or yields of such reactions, so that this branch might be termed magnetokinetics. In this paper we will review some of our recent work demonstrating how this technique can be used for probing the dynamics of short-lived intermediates in photoinduced electron transfer reactions. In our study spin-orbit coupling (SOC) provides a specific handle for applying the magnetokinetic approach.

Let us first consider the typical stages of charge transfer and separation during a photoinduced electron transfer reaction (cf. scheme 1. While this scheme is fairly general, the charges of the reactants correspond to the particular situations investigated by us). For a random distribution of reactants in a solution one first requires formation of encounter pairs between donor and acceptor molecules, one of them being electronically excited. Electron transfer, then, will produce a primary redox pair, in

\footnotetext{
* For correspondence
} 


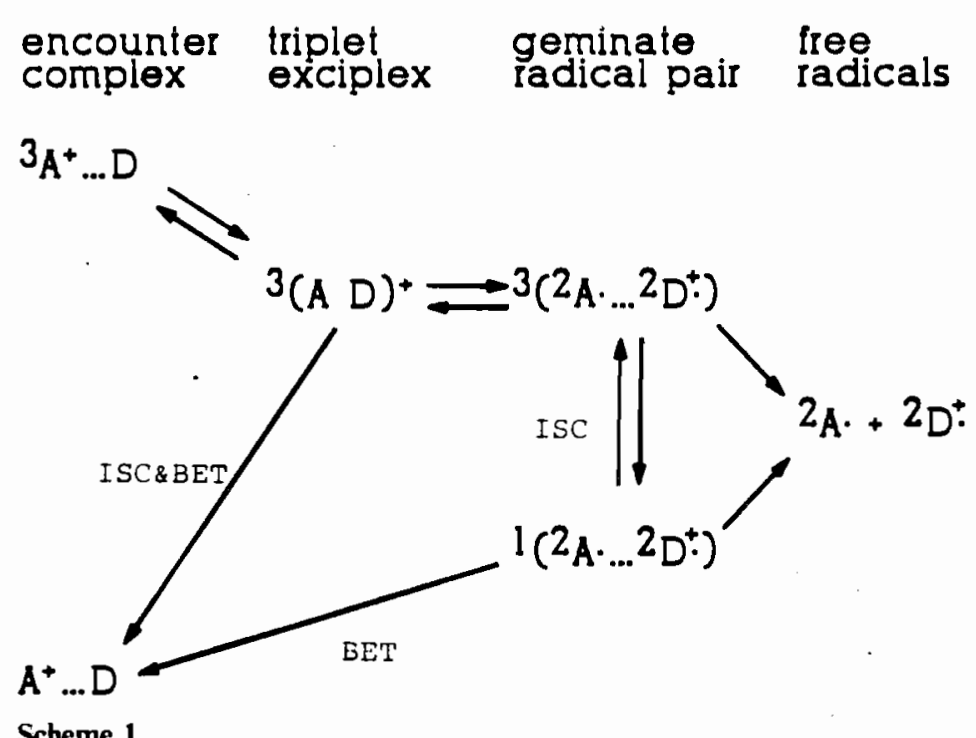

close contact, with perhaps still considerable intermolecular electronic interaction and charge delocalization. This species will be called an exciplex. It can dissociate to a solvent-shared radical pair, now with the transferred electron definitely localized on the electron acceptor. Controlled by diffusion the radicals of the pair may undergo successive re-encounters with their initial partner and during that stage the pair will be called a geminate or correlated radical pair. After they have finally separated they become uncorrelated free radicals, and it is at this stage that the products of electron transfer are fully available.

Before this stage is reached, however, fast electron back-transfer regenerating the initial reactants in their ground states, either from the stage of the exciplex or from the stage of the correlated radical pair, can diminish the desired high yield of product formation. Hence it will be important to understand the dynamics of the intermediates and to have methods available for measuring it. Magnetokinetics can serve this purpose if spin changes are involved in the reaction scheme. This is the case if, for example, the photoreactive state is a triplet state and if the backward electron transfer goes to the singlet ground state (scheme 1). In the forward transfer the primary products will then originate with parallel spins and a spin reorientation is needed while backward electron transfer to the singlet occurs or before it takes place. If one knows the mechanisms of spin-realignment in the intermediates and how they are affected by an external magnetic field it is possible to determine their dynamics from the magnetic field dependence observed for the final product yield which is relatively easy to measure on a much longer time scale.

\section{The role of exciplex and radical pair intermediates in photo electron transfer reactions}

Our first example (Steiner and Haas 1991) will be concerned with the electron transfer reaction between aniline (An, electron donor $D$ ) and photoexcited methylene blue triplet $\left({ }^{3} \mathrm{MB}^{+}\right.$, electron acceptor $\left.{ }^{3} \mathrm{~A}^{+}\right)$. The dye triplet is rapidly quenched by this reaction and the yield of free radicals produced is close to unity. This means that 
backward electron transfer in the primary redox products is very inefficient. The reason for this is that it is spin forbidden and interactions that could induce a spin conversion during the exciplex or the geminate radical pair stage are obviously too weak. The situation changes if aniline is replaced by the heavy atom substituted donor $p$-iodoaniline ( $p$-I-An). In this case SOC becomes so strong that most of the primary redox pairs find their way to the singlet ground state before they can diffuse apart to yield free radicals. This effect is enhanced by increasing the solvent viscosity.

Remember at this point that in introducing a heavy atom substituent the idea is to provide for an efficient process of spin conversion in the primary redox intermediates, a process that can be modified by a magnetic field so that specific information on the dynamics of these short-lived intermediates is transformed into a magnetic field effect on the yield of long-lived free radicals. How this magnetic field effect is borne out in the transient signals is shown in figure 1. This radical yield reducing effect is specific to the system with the heavy atom substituted donor. No radical yield reduction by a magnetic field effect has been detectable with the system ${ }^{3} \mathrm{MB}^{+} / \mathrm{An}$. For the system ${ }^{3} \mathrm{MB}^{+} /$dimethylaniline in methanol/ethylene glycol mixtures it was possible to detect increases of the radical yield on the order of $1 \%$ at low magnetic fields. These effects may be attributed to the hyperfine coupling mechanism which is stronger in the system with the $\mathrm{N}$-methylated donor. The effects are of opposite sign and much weaker than the heavy atom dependent magnetic field effects described and analyzed in this paper.

The field dependence of the relative magnetic field effect is presented in some detail

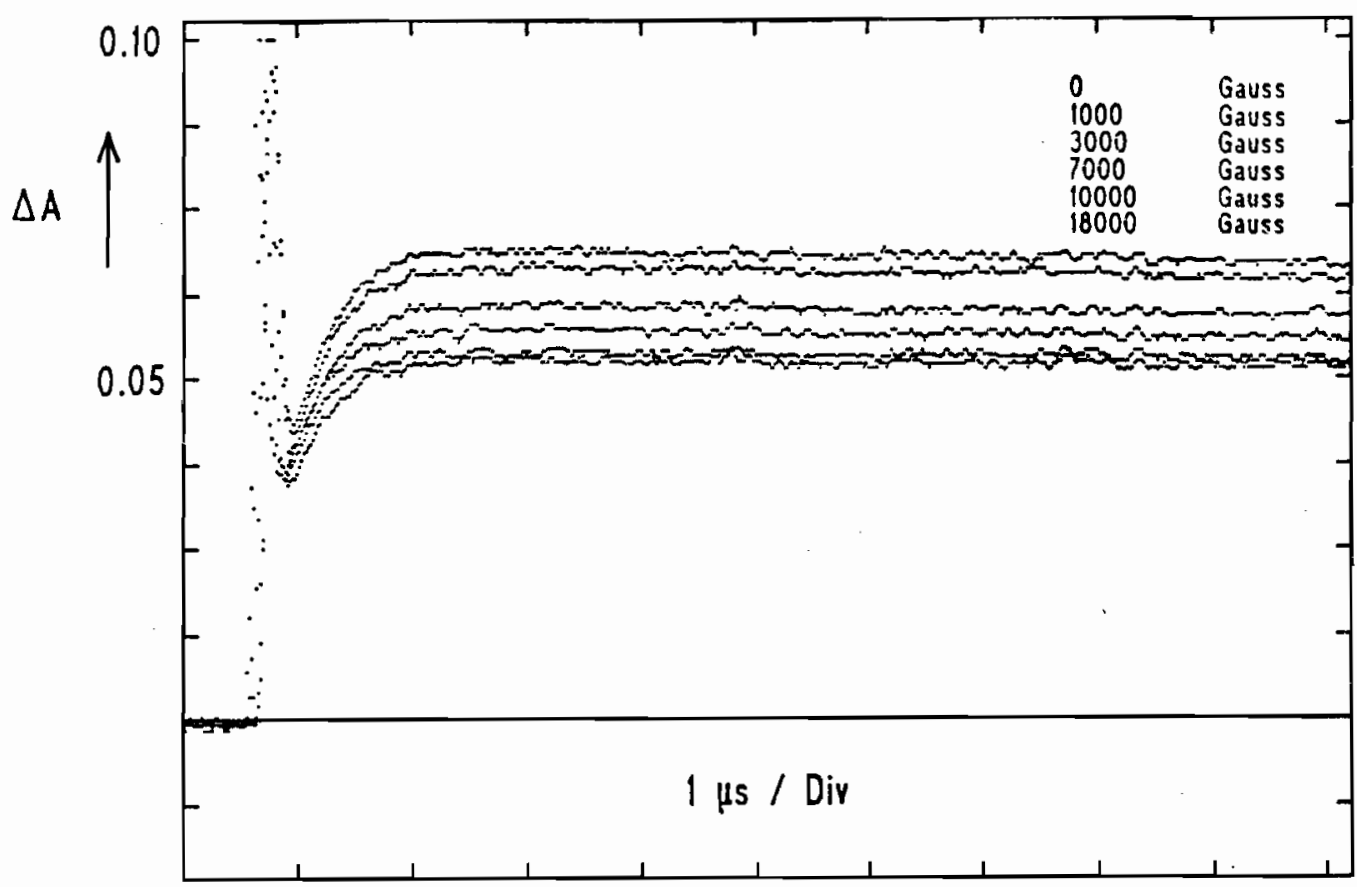

Figure 1. Transient absorption signals at $840 \mathrm{~nm}$ observed by laser flash spectroscopy on quenching ${ }^{3} \mathrm{MB}^{+}$by $p-\mathrm{I}-\mathrm{An}$ in methanol buffered at $\mathrm{pH}^{*}=8 \cdot 6$. The rapidly decaying initial part is due to $T-T$ absorption of ${ }^{3} \mathrm{MB}^{+}$, the rising and persistent part of the signal due to the semiquinone radical $\mathrm{MBH}{ }^{ \pm}$formed by protonation of $\mathrm{MB}$ - radicals resulting from the quenching reaction but not observable at this wavelength. The figure demonstrates how the yield of radicals is decreased when applying external magnetic fields. 


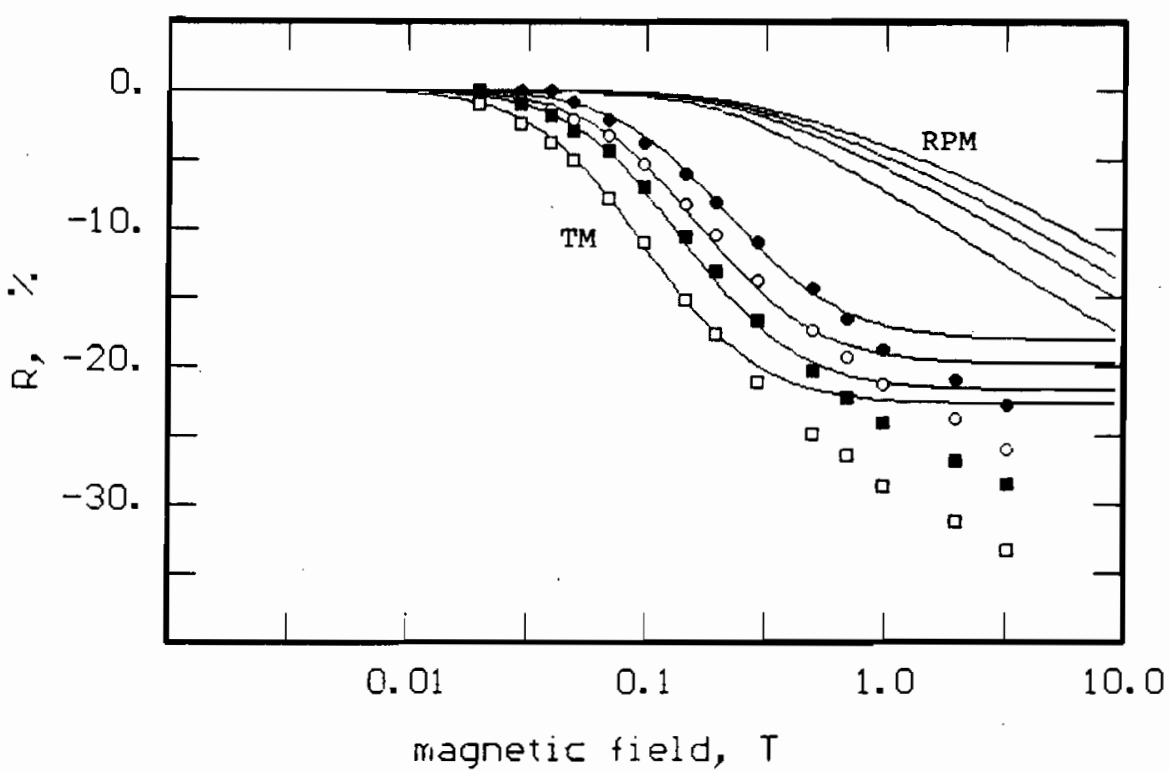

Figure 2. Relative magnetic field effect $R$ on free radical yield in reaction of ${ }^{3} \mathrm{MB}^{+}$with p-I-An. Experimental valies for $\mathrm{MeOH} /$ ethylene glycol mixed solvents with (O) $100 \%,(\mathrm{O})$ $90 \%$, (口) $80 \%,(\square) 60 \% \mathrm{MeOH}$. The set TM of curves results from model calculations using the triplet mechanism only, the set RPM from calculations employing the radical pair mechanism only.

in figure 2. Its onset is typically in the range of some 20 to $30 \mathrm{mT}$, the steepest slope at about $0.2 \mathrm{~T}$. Increasing the solvent viscosity enhances the magnetic field effect and shifts the onset to lower fields. At 3.3 T, the highest field applied, the magnetic field effect corresponds to a decrease of free radical yield between 22 and $32 \%$.

In the analysis of the magnetokinetic information let us consider the contribution of the correlated radical pairs first (cf. figure 3). From the forward electron transfer reaction these originate with parallel spins, i.e. in an overall triplet state. In the magnetic fields, where an effect on the free radical yield becomes detectable, the Zeeman splitting of the triplet components is fairly strong and $T_{+}$and $T_{-}$can be considered as kinetically isolated during the lifetime of the correlated radical pair. The spin substates $T_{0}$ and $S$ remain degenerate. Transitions between them are induced by different Larmor precession frequencies of the electron spin at the two radicals caused by different hyperfine fields and different $g$-factors. In the present system a major difference of $g$-factors arises through the strong SOC in the donor radical. The $\Delta g$-dependent $T_{0}-S$ transition frequency increases linearly with external field strength.

$$
\Delta \omega=\gamma e \Delta g B_{0} / 2,
$$

with $y e$ the gyromagnetic ratio of the electron, $\Delta g$ the difference of radical $g$-factors and $B_{0}$ the magnetic induction of the external magnetic field.

Radical pairs in an overall singlet spin state will recombine with high probability on subsequent re-encounters. In this way the magnetic field effect enhances the efficiency of geminate backward electron transfer and decreases the yield of free radicals. The theoretical formalism to describe this effect quantitatively is well documented in the literature (cf. Steiner and Ulrich 1989 or Salikhov et al 1984, and 


\section{Triplet Mechanism}

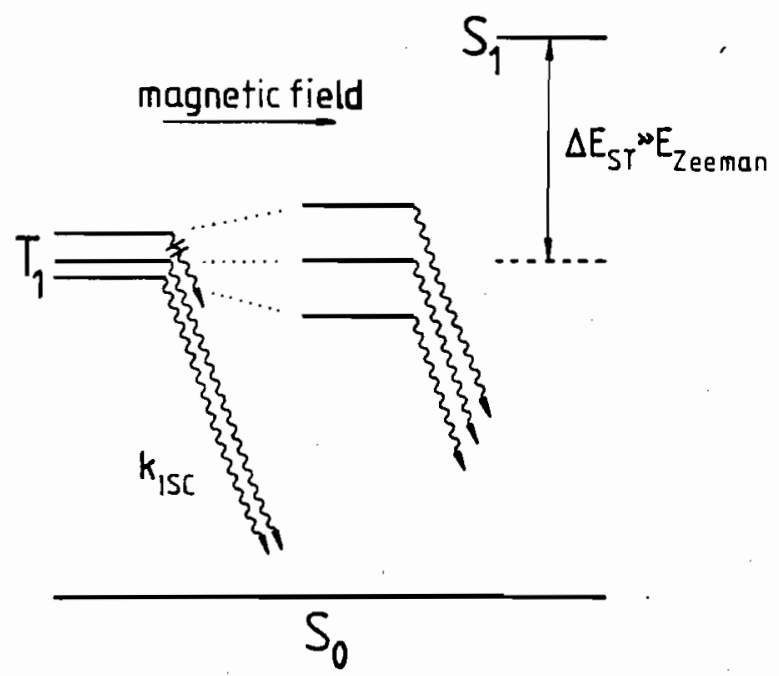

Radical Pair Mechanism

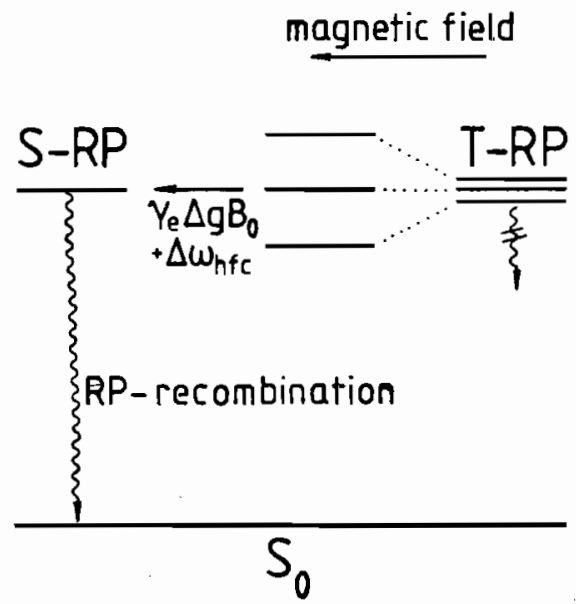

Figure 3. Two cases of SOC induced magnetokinetic effects. In the radical pair mechanism recombination to a singlet product is only possible from the radical pair singlet substate. A magnetic field may support recombination of triplet radial pairs by $\Delta g$-dependent mixing of radical pair $T_{0}$ and $S$ spin substate. The triplet mechanism, applying for example to heavy atom containing triplet exciplexes, requires that fast direct SOC-induced transitions from triplet substates to the singlet ground state can occur. Due to symmetry selection rules SOC does not affect the triplet substates equally and they decay at different rates. In a magnetic field the zero-field triplet substates are mixed. Thereby their kinetic distinction is lost. Increasing uniformity of triplet sublevel decay enhances the overall efficiency of the $T-S_{0}$ process. The excited singlet state is not involved in case of this mechanism because the $T / S$ energy splitting is too wide. Note that the splitting of the triplet levels is represented in a strongly enlarged scale as compared to the $T / S_{0}$ energy gap.

references given there). The problem adopts an analytically tractable form if only the mixing of two radical pair spin substates $\left(T_{0}\right.$ and $S$ ) has to be considered and there are no Coulombic forces between them. The free radical yield may be calculated as a function of the magnetic field on the basis of the following set of parameters (Pedersen 1977; Purtov and Salikhov 1980).

$D \quad$ the sum of diffusion coefficients of the radicals;

a the reaction distance;

$\lambda s$ the reaction probability of a radical pair when having an encounter in the singlet spin substate;

$\Sigma_{\mathrm{HFC}}$ the root mean square deviation of longitudinal hyperfine field differences between the two radicals for all possible nuclear spin configurations;

$\Delta g \quad$ the difference of $g$-factors of the two radicals.

In addition, the effect of electronic interaction in the radical pair can be accounted for in the dynamic theory (Vollenweider and Fischer 1988) using the parameters $J_{0}$ and $r_{J}$ for an exchange interaction potential decaying exponentially with radical separation $r$ :

$$
J(r)=J_{0} \exp \left(-r / r_{J}\right)
$$


In figure 2 are shown the results of numerical calculations of the radical pair type magnetic field effect. The physically most reasonable parameter values have been used. It should be noted, however, that even with a physically unreasonably extreme choice of parameters the model calculations fail to account for the experimentally observed magnetic field dependence. Therefore it is necessary to consider also a possible magnetic field dependence of backward electron transfer in the triplet exciplex stage preceding the correlated radical pair (cf. scheme 1).

In the exciplex the electronic interaction is much stronger than in a solvent-shared radical pair. Thus the energy separation $T_{\mathrm{Ex}} / S_{\mathrm{Ex}}$ is much larger than can be bridged by a magnetic coupling. As opposed to the radical pair situation, in the exciplex the heavy atom substituent is an integral part of an electronically excited supermolecule. Spin-forbidden back transfer of the electron just corresponds to the radiationless ISC process of this species and it is strongly enhanced by SOC at the heavy atom centre. It is well known that SOC in molecules depends on the orientation of electronic spin relative to the molecular frame (McGlynn et al 1969). For the present system is has been shown (Steiner 1981) that the heavy atom effect will be weak if the electronic spin is parallel to the aromatic plane of the donor. We call this substate $T_{z}$ and note that, since its direct ISC is slow, it contributes predominantly to the yield of radicals formed upon dissociation of the exciplex. In a magnetic field the spin undergoes rapid Larmor precession and quickly reorients with reference to the molecular frame. This corresponds to a mixing of substate $T_{z}$ with $T_{x}$ and $T_{y}$ in the new eigenstates of the spin Hamiltonian with increasing contributions of Zeeman interaction. It can be shown that with the radiationless decay rate distributed more evenly over the triplet substates, the overall decay yield to the ground state increases, and hence the free radical yield decreases. This mechanism of a magnetokinetic effect is called the triplet mechanism. Its quantitative theory has been developed some time ago (Atkins and Evans 1974; Steiner 1981; Serebrennikov and Minaev 1987). Parameters entering into the calculation are

$k_{\mathrm{ER}}$ the rate constant of dissociation into radicals;

$k_{\text {ISC }} \quad$ the rate constant of substate selective intersystem crossing;

$D_{r}$ the rotational diffusion constant of the triplet exciplex.

In figure 2 there is also shown a fit of the experimental magnetic field effect by the triplet mechanism. The fit is excellent up to about $0.4 \mathrm{~T}$ but fails at higher fields where it predicts a saturation. If, however, we link together the triplet mechanism in the triplet exciplex and the $\Delta g$-type radical pair mechanism in the correlated radical pair originating from dissociation of the triplet exciplex the theoretical calculations yield an excellent fit over the whole field region investigated for all four solvents of different viscosity (cf. figure 4). The parameters of the radical pair mechanism have not been particularly optimized; experimental values have been used where available, or the physically most reasonable estimates otherwise. The successful modelling of the experimental results by the combination of both mechanisms provides convincing evidence that the existence of both intermediates of free radical formation, exciplex and correlated radical pair, is in fact borne out by the magnetokinetic measurements.

The parameter values determined for the triplet exciplex dynamics for solvent mixtures ( $\mathrm{MeOH} /$ ethylene glycol) of different viscosity are represented in figure 5 . The rate constant of dissociation is by about a factor of 10 slower than estimated by the Eigen equation (Eigen 1954) for dissociation of an unbound pair of non-interacting 


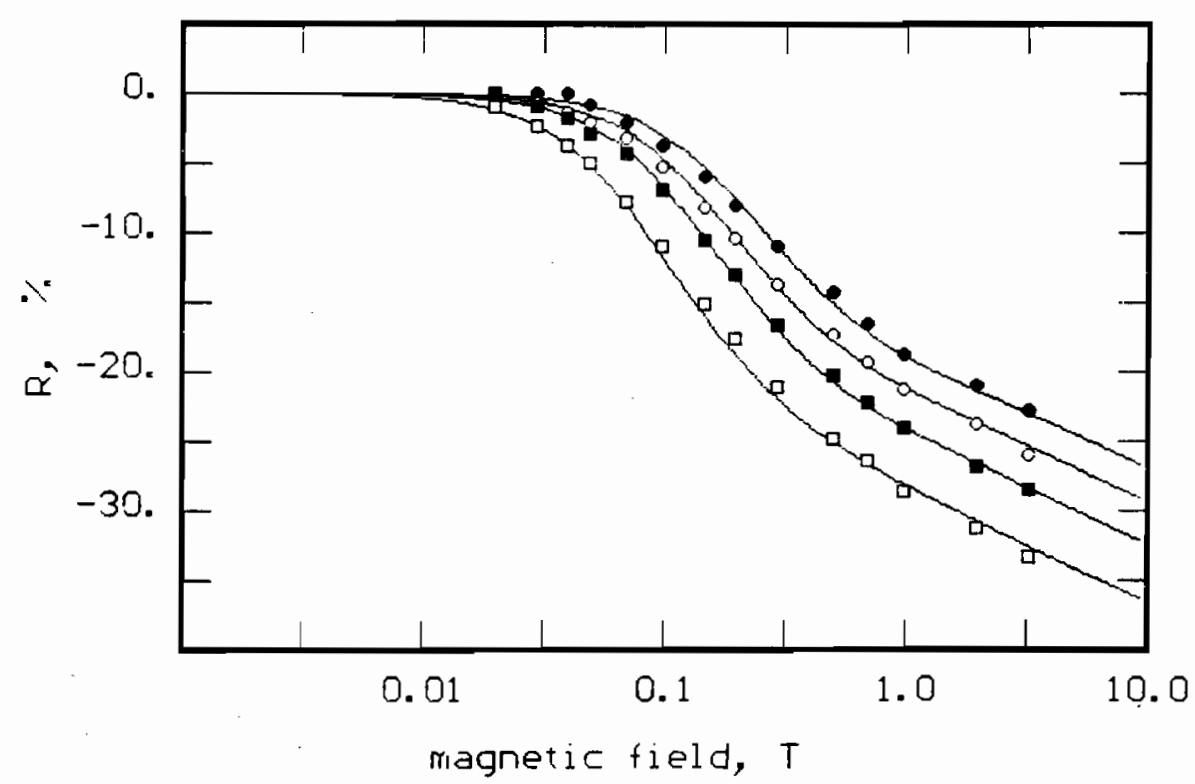

Figure 4. Modelling of the experimental magnetic field effect (cf. figure 2) by a combination of triplet and radical pair mechanism. The parameters used for the triplet mechanism in the solvents of different viscosity are shown in figure 5. The parameters of the radical pair mechanism are $\lambda_{s}=1, a=0.7 \mathrm{~nm}, \Sigma_{\mathrm{HFC}}=0.6 \mathrm{mT}, \sigma=0.01 \mathrm{~nm}, \Delta g=0.015, D=(2.15,1.56$, $1 \cdot 12,0 \cdot 6) \times 10^{-5} \mathrm{~cm}^{2} \mathrm{~s}^{-1}$ for $100,90,80,60 \% \mathrm{MeOH}$, respectively.

particles. It is seen to vary linearly with the inverse of solvent viscosity. The slope, depending somewhat on the model assumptions is in very good agreement with what Weller (1982) has evaluated for singlet exciplexes.

The rotational diffusion constant $D_{r}$ is almost proportional to the inverse of the solvent viscosity. Applying Debye's expression for the rate constant of rotational diffusion one can estimate an effective hydrodynamic radius of the exciplex of about $0.36 \mathrm{~nm}$ which fits rather well with what one expects from a molecular model. The rate constant of ISC in the exciplex has the weakest dependence on solvent viscosity, as expected. The value of about $3 \times 10^{10} \mathrm{~s}^{-1}$ corresponding to $30 \mathrm{ps}$ lifetime is very fast for an ISC process, but becomes understandable by the strong internal heavy atom effect of the iodine substituent.

\section{Spin memory in Ru(III) complex/methylviologen radical redox pair}

Our second example of applying SOC-induced magnetokinetic effects for the study of photoelectron transfer mechanisms is concerned with the well-known reaction between photoexcited $\mathrm{Ru}$-trisbipyridyl $\left(\mathrm{Ru}[\mathrm{bpy}]_{3}^{++}\right)$and methylviologen $\left(\mathrm{MV}^{++}\right)$ (Moradpour et al 1978; Kalyanasundaram 1982; Juris et al 1988) (figure 6). Taking into account the repulsive Coulombic interaction between the two species the rate constant of the photoelectron transfer reaction of about $5 \times 10^{8} \mathrm{M}^{-1} \mathrm{~s}^{-1}$ corresponds to an almost diffúsion controlled reaction. However, the efficiency of free radical formation of $\mathrm{MV}^{+}$is only of the order of $25 \%$ (Hoffman 1988) indicating a $75 \%$ efliciency of fast backward electron transfer before the primary redox pair can fully separate. The photoreactive state of the Ru complex may be characterized as a MLCT 

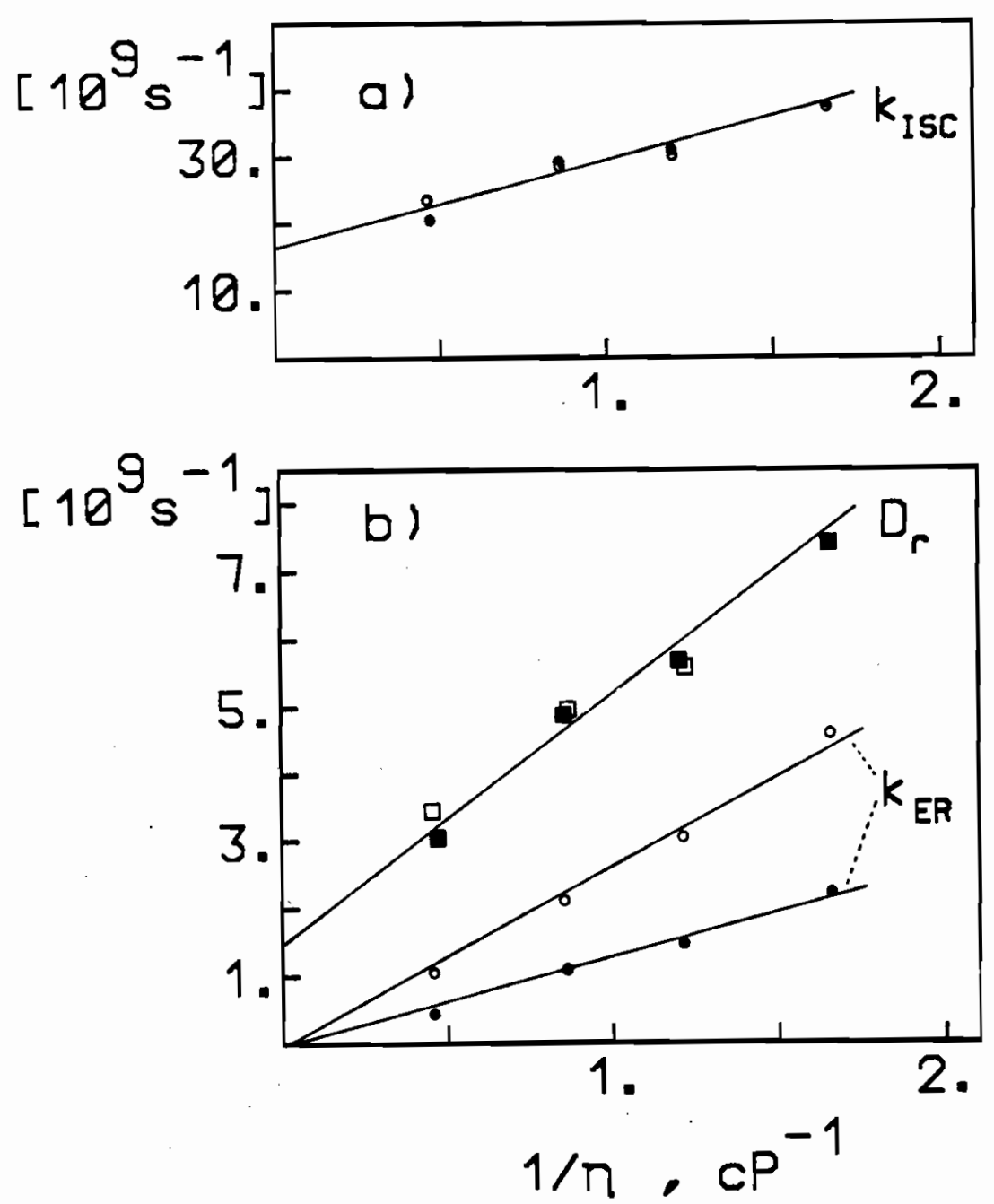

Figure 5. Viscosity dependence of the parameters $k_{\mathrm{ISC}}, k_{\mathrm{ER}}, D_{r}$ determined for the triplet exciplex ${ }^{3}(\mathrm{MB} . . \mathrm{p}-\mathrm{I}-\mathrm{An})^{+}$. Closed symbols: results obtained from the fits assuming irreversible exciplex dissociation, open symbols: for reversible exciplex dissociation assuming that the overall probability of geminate reformation of the exciplex is $50 \%$.

triplet state (Krausz and Ferguson 1989). Accordingly the primary redox pair, too, should originate with triplet spin correlation and the backward electron transfer yielding the singlet ground state reactants should be spin-forbidden. Investigators of this system have not paid much attention to this aspect, so far. Actually a general opinion is that spin memory cannot be of importance in systems with so strong a SOC as in the trivalent Ru-complex. Our magnetokinetic studies with this system (Steiner et al 1989; Wolff and Steiner 1990; Steiner and BürBner 1990) have revealed, however, that some spin-memory is detectable even with such a system. It causes a magnetic field dependence of the yield of free radicals.

It should be mentioned that magnetic field effects in this system have been also reported by Ferraudi and Arguello (1988) and by Kiwi (1983). The former offered an explanation basically different from ours, but not in accord with all experimental facts. In Kiwi's work the $\mathrm{Ru}[\mathrm{bpy}]_{3}^{++} / \mathrm{MV}^{++}$system was used to sensitize $\mathrm{H}_{2}$ 


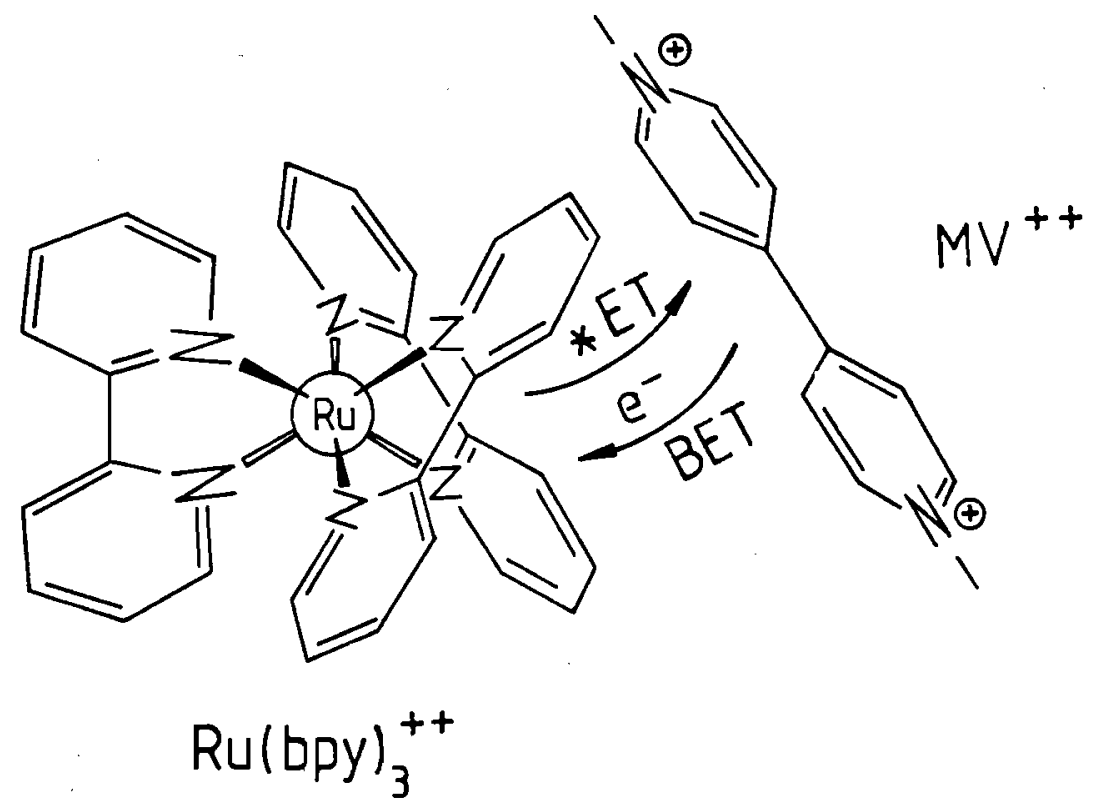

Figure 6. Quenching of photoexcited $\mathrm{Ru}(\mathrm{bpy})_{3}^{++}$by $\mathrm{MV}^{++}$occurs by fast electron transfer $\left({ }^{*} \mathrm{ET}\right)$ generating a $\mathrm{Ru}(\mathrm{bpy})_{3}^{+++} / \mathrm{MV}^{+}$redox pair, which undergoes fast backward electron transfer (BET) to regenerate the ground state reactants (ca. $75 \%$ ) in competition with cage escape yielding free redox products (ca. $25 \%$ ).

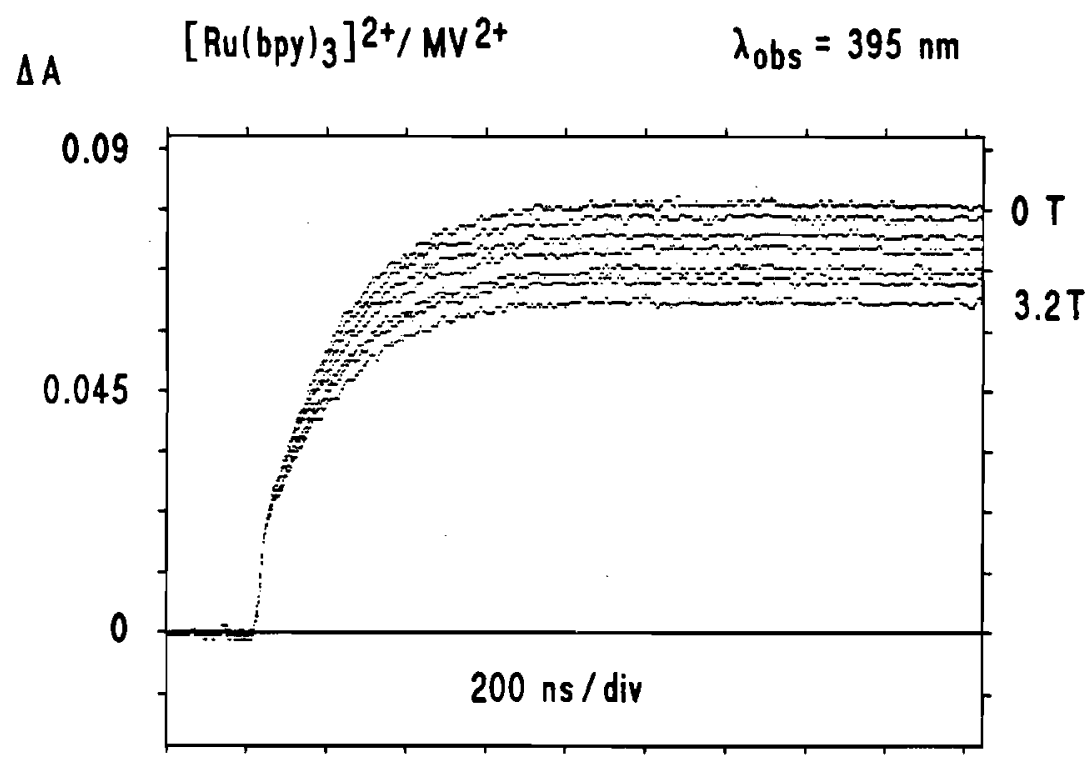

Figure 7. Magnetic field effect on radical yield in the quenching of photoexcited $\mathrm{Ru}(\mathrm{bpy})_{3}^{++}$ by $\mathrm{MV}^{++}$. The transient absorption signals were observed at $395 \mathrm{~nm}$. Magnetic fields (from top to bottom) $0 \cdot 0,0 \cdot 5,1 \cdot 0,1 \cdot 5,2 \cdot 0,2 \cdot 5,3 \cdot 2$ Tesla.

evolution at the surface of semiconductor particles. A magnetic field effect on the rate of $\mathrm{H}_{2}$-production was attributed to surface recombination of radicals.

In figure 7 we give a demonstration of the magnetic field effect as observed in our laboratory by nanosecond laser flash spectroscopy. The time-resolved rise of absorption is due to the formation of the $\mathrm{MV}^{+}$radical. As can be seen the yield of 
radicals decreases with increasing magnetic field. The quenching rate constant is independent of the field and the magnetic field effect is due to a change in the yield of cage escape from the primary redox pair.

It can be explained and analyzed in terms of magnetic field enhanced backward electron transfer. In principle this effect is of the same type as the $\Delta g$-mechanism in a radical pair. It requires, however, a specific theoretical treatment that properly takes into account the SOC at the Ru centre (Steiner et al 1989). The essential difference with respect to the previous case is that the energy separation between the singly-occupied frontier molecular orbitals (this energy separation results from a weak trigonal ligand field splitting of the $\mathrm{Ru} d$-orbitals) is of the same order of magnitude as the SOC energy between them. As a consequence a strong mixing of different spin-orbit states takes place and the selection rules for the reactivity of the redox pair's effective spin states are not as simple as in a normal radical pair because the effective spin is not conserved in the backward electron transfer reaction. We have worked out a suitable dynamic treatment for this new magnetokinetic situation (Steiner and Bürßner 1990) which allowed us to analyze the observed magnetic field dependence in terms of the following parameters:

$k_{\text {ce }} \quad$ the rate constant of cage escape

$k_{\text {bet }}$ the rate constant of hypothetically spin-allowed backward electron transfer

$T_{s} \quad$ the time constant of spin relaxation in the $\mathrm{Ru}(\mathrm{III})$ complex. $T_{1}=T_{2}\left(\equiv T_{s}\right)$ was assumed.

g the $g$-tensor of the Ru(III) complex.

Furthermore the initial population of the four lowest spin-orbit substates has to be fixed.

Using the known experimental values of the $g$-tensor (DeSimone and Drago 1970) of the oxidized complex $\left[\mathrm{Ru}(\mathrm{bpy})_{3}\right]^{+++}$and fixing the rate constant of cage escape by the Eigen-Debye equation the other parameters were determined from a fit of the experimental magnetic field dependence of the radical yield (cf. figure 8).

The important result is that only with a very short spin relaxation time $T_{s}$ of about 25 ps the experimental curve can be reasonably fitted. We had expected that rotation of the Ru-complex, which has an extremely anisotropic $g$-tensor, should be the dominating mechanism for spin-relaxation in liquid solution. The value actually found is more than ten times shorter than explicable in terms of such a rotational diffusion mechanism. There is, however, independent experimental evidence of the extremely fast electron spin-relaxation in $\mathrm{Ru}(\mathrm{bpy})_{3}^{+++}$. This comes from NMR spectra measured with $\mathrm{Ru}(\mathrm{bpy})_{3}^{+++}$-type paramagnetic complexes in solution at room temperature (DeSimone and Drago 1970). The line width exhibited by these NMR spectra places the electron spin relaxation time well below 100 ps. It may be anticipated that our magnetokinetic approach as described in this paper opens up a new experimental access to investigate ultra-fast electron spin relaxation of paramagnetic complexes in liquid solution.

Another result to be emphasized is the high value of $10^{11} \mathrm{~s}^{-1}$ for the rate constant of spin-allowed backward electron transfer. It is about 7 times faster than the rate constant estimated in the literature (Hoffmann 1988) without any spin-considerations. 


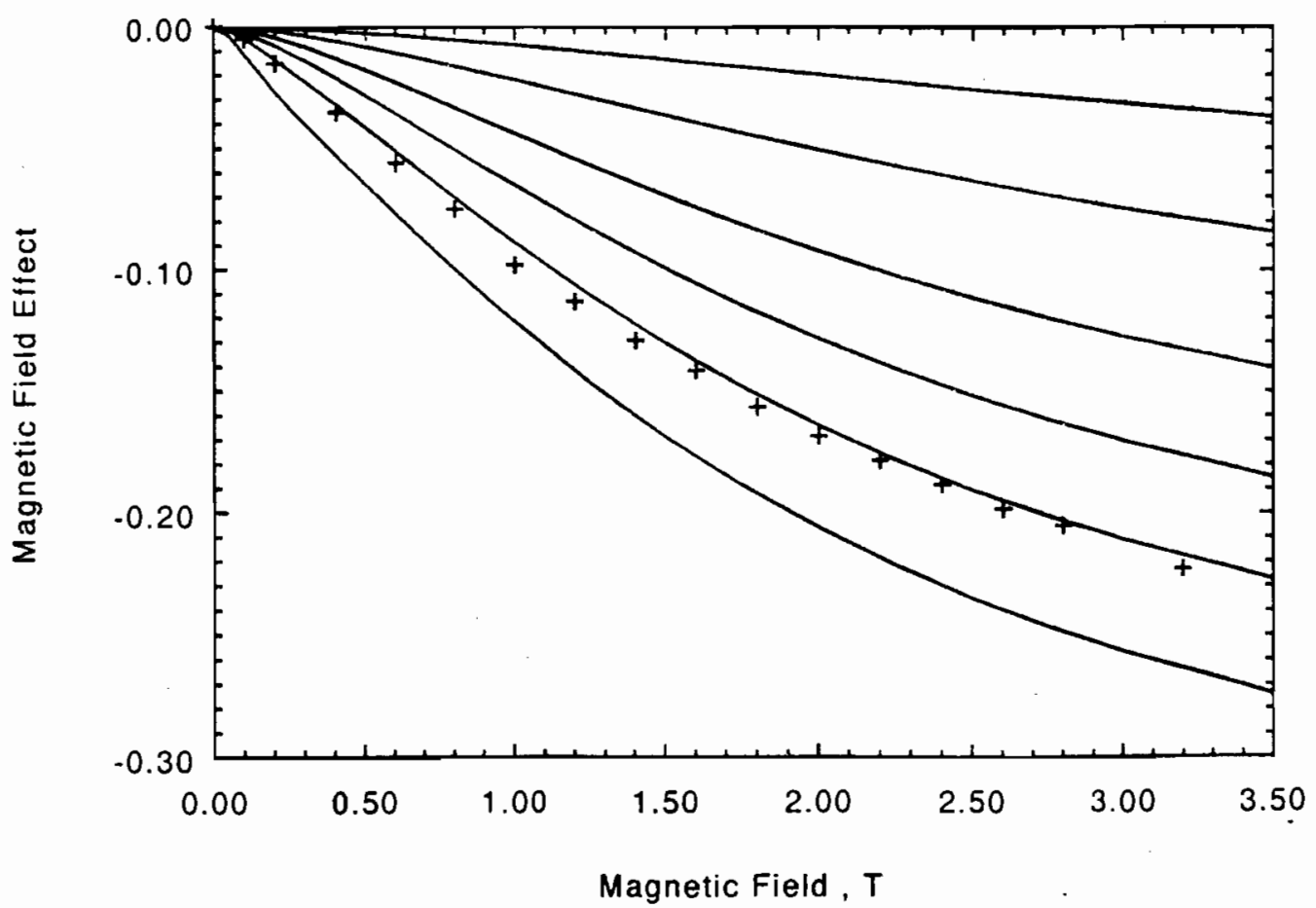

Figure 8. Observed $(+)$ and calculated (solid lines) magnetic field effects on free radical formation in the $\mathrm{Ru}(\mathrm{bpy})_{3}^{++} / \mathrm{MV}^{++}$photoreaction. It was assumed that only formal triplet substates of the effective spin of the primary redox pair were populated in the photoinduced forward electron transfer. The values used for the other model parameters were: $k_{\mathrm{ce}}=5 \mathrm{~ns}^{1}$, $1 / T_{s}$ was varied along the series of curves as (from top to bottom) $200,120,80,60,45,30 \mathrm{~ns}^{-1}$. The value of $k_{\text {bet }}$ was adjusted for each curve such that in zero field an absolute yield of free radicals of $25 \%$ was obtained. The actual values of $k_{\text {bet }}$ thus employed were (from top to bottom) $76,89,108,128,155,201 \mathrm{~ns}^{-1}$.

\section{Acknowledgements}

This research was supported by the Deutsche Forschungsgemeinschaft and by the Fonds der Chemischen Industrie.

\section{References}

Atkins P W and Evans G T 1974 Mol. Phys. 271633

DeSimone R E and Drago R S 1970 J. Am. Chem. Soc. 922343

Eigen M $1954 Z$. Phys. Chem. 1176

Ferraudi G and Arguello G A 1988 J. Phys. Chem. 921846

Hayashi H 1990 in Photochemistry and photophysics (ed.) J F Rabek (CRC Press: Boca Raton) vol. 1, p. 59 Hoffman M Z 1988 J. Phys. Chem. 92 3458; cf. also 1991 J. Phys. Chem. 952606 (erratum) Juris A, Balzani V, Barigelletti F, Campagna S, Belser P and von Zelewsky A 1988 Coord. Chem. Rev. 8485 Kalyanasundaram K 1982 Coord. Chem. Rev. 46159

Kiwi J 1983 J. Phys. Chem. 872274 
Krausz E and Ferguson J 1989 Prog. Inorg. Chem. 37293

McGlynn S P, Azumi T and Kinoshita M 1969 Molecular spectroscopy of the triplet state (Englewood Cliffs, NJ: Prentice-Hall)

Moradpour A, Amouyal E, Keller P and Kagan H 1978 Nouv. J. Chim. 2547

Pedersen J B 1977 J. Chem. Phys. 674097

Purtov P A and Salikhov K M 1980 Theor. Exp. Chem. 16413

Salikhov K M, Molin Yu N, Sagdeev R Z and Buchachenko A L 1984 Spin polarization and magnetic effects in radical reactions (Eisevier: Amsterdam)

Serebrennikov Yu A and Minaev B F 1987 Chem. Phys. 114359

Steiner U E 1981 Ber. Bunsenges. Physik. Chem. 85228

Steiner U E and BürBner D 1990 Z. Phys. Chem. 169159

Steiner U E and Haas W 1991 J. Phys. Chem. 951880

Steiner U E and Ulrich T 1989 Chem. Rev. 8951

Steiner U E, Wolff H -J, Ulrich T and Ohno T 1989 J. Phys. Chem. 935147

Vollenweider J -K and Fischer H 1988 Chem. Phys. 124333

Weller A 1982 Z. Phys. Chem. 130129

Wolf H -J and Steiner U E 1990 Z. Phys. Chem. 169147 